\title{
Limitations of the IAU2000 nutation model accuracy due to the lack of Oppolzer terms of planetary origin
}

\author{
José M. Ferrándiz ${ }^{1}$, Juan F. Navarro ${ }^{1}$, M. C. Martínez-Belda ${ }^{1}$, Alberto Escapa ${ }^{2,1}$, and Juan Getino ${ }^{3}$ \\ 1 Dept. of Applied Mathematics, University of Alicante, PO Box 99, 03080 Alicante, Spain \\ e-mail: jm. ferrandiz@ua.es \\ 2 Dept. of Aerospace Engineering, University of León, 24071 León, Spain \\ 3 Dept. of Applied Mathematics, University of Valladolid, 47011 Valladolid, Spain
}

Received 21 March 2017 / Accepted 4 July 2018

\begin{abstract}
Context. The current IAU2000 nutation model performed different approximations, one of them being that the Oppolzer terms associated to the planetary perturbations of the nutations were assumed to be smaller than $5 \mu$ as and thus were neglected. At present, the uncertainties of the amplitudes of individual components of the observed nutations are better, and the conventional nutation model does not fit the accuracy requirements pursued by the International Astronomical Union (IAU) and the International Association of Geodesy (IAG).

Aims. The objective of this work is to estimate the magnitude of the lacking Oppolzer terms of the planetary nutations and find out whether they are still negligible or not.

Methods. The Oppolzer terms resulting from the direct and indirect planetary perturbations of the Earth's rotation have been computed for a two-layer Earth model following the Hamiltonian theory of the non-rigid-Earth.

Results. The planetary Oppolzer terms for the non-rigid Earth are not really negligible as believed, and some of them have amplitudes larger than $10 \mu$ as, therefore significantly above the current level of uncertainty of individual harmonic constituents.

Conclusions. In the short term, the IAU2000 nutation model must be supplemented with suitable corrections accounting for those missing components; its planetary component must be thoroughly revised in the medium term.
\end{abstract}

Key words. astrometry - celestial mechanics - Earth - ephemerides - reference systems

\section{Introduction}

Resolution B1.6 of the twenty fourth General Assembly of the International Astronomical Union (IAU) endorsed the nonrigid-Earth solution MHB2000 (Mathews et al. 2002) as the IAU2000A nutation model, which is concisely described in paragraph [8] of Herring et al. (2002) as "generated by the convolution of the transfer function from Mathews et al. (2002) with the rigid-Earth nutation series REN2000" (Souchay et al. 1999). However, the current situation is not so simple: as it happens, with its underlying rigid-Earth rotation theory, the IAU2000 theory covers two different groups of terms. The first one has 678 terms and accounts for the direct lunisolar perturbations (excluding planetary perturbations of the Moon and Sun orbits), which are those of largest magnitude and constitute the nutation series MHB2000 in a strict sense; they depend solely on the five Delaunay arguments $\left(l, l^{\prime}, F, D, \Omega\right)$ used by Kinoshita (1977). The second block is formed by 687 terms corresponding to the direct and indirect planetary perturbations, which are directly taken from the underlying rigid theory, and have amplitudes smaller than 0.5 mas (Herring et al. 2002); the planetary nutations are expressed in a different set of arguments, namely the eight mean longitudes of the planets and the precession parameter $p_{\mathrm{A}}$. The developments of the arguments in both sets are displayed in Sect. 3.7 of the IERS Conventions (2010) published by the International Earth Rotation and Reference Systems Service (IERS).

The details are clearly documented in the computer programs used for the application of the theory. Thus, in the original routine MHB_2000.f, used for the computation of IAU $2000^{1}$, the subroutine out_plan_nut that calculates the planetary nutations specifies the following "APPROXIMATIONS: The Oppolzer terms have not been added (should be $<0.005$ mas), and Contributions from a non-rigid Earth have not been computed. For many of these terms the contribution arises from the perturbation of the Earth's orbit and therefore there will be not deformation effects". The figure of $5 \mu$ as was the threshold established by Resolution B4 of the IAU 1997 General Assembly for the truncation of the new rigid-Earth nutation series whose development was urged in it.

Moreover, the amplitudes of the lunisolar components of the underlying rigid-Earth nutation model in that routine - used to apply the MHB2000 transfer function - have been taken from the so-called series SKRE1997 (Souchay \& Kinoshita 1997), while those for the planetary nutations - which have not been modified - have been extracted from the previous solution KS1990 (Kinoshita \& Souchay 1990). Unlike the lunisolar terms, the IAU2000 built-in planetary terms have not been published as tables or attached electronic files in any of the articles that developed the MHB2000 theory. It is mandatory to turn to the aforementioned software for the computation of nutations or, for example, to the well-known Standards of Fundamental Astronomy (SOFA) library (Hohenkerk et al. 2010) for their accurate identification; planetary nutations are also available as

1 Still available at http://wWw-gpsg.mit.edu/ tah/mhb2000/ MHB_2000.f 
American Standard Code for Information Interchange (ASCII) tables in Kaplan (2005) or at the web site of the IERS Conventions Center ${ }^{2}$.

In summary, although it is usually said that the IAU2000 nutation theory is the result of applying the MHB2000 transfer function to REN2000 to take into account the non-rigidity effects on the nutations, the truth is that around half of its terms are actually rigid nutations, since the transfer function was not applied to them. Moreover, those small planetary terms are reduced to the so-called Poisson terms, just because the rigid-Earth Oppolzer terms - of much smaller magnitude - have not been taken into account either. In other words, the successive evolutions of Kinoshita's theory just computed the perturbations exerted by the planets for the nutations of the angular momentum axis, that is, Poisson terms. They were grouped into two separated blocks: the direct and indirect planetary nutations. This splitting corresponds to the source of the planetary perturbation as derived from the semi-analytical theory of the Moon motion ELP, detailed for example in Chapront-Touzé \& Chapront (1983a). The differences in the nutations of the figure axis and angular momentum axis (Oppolzer terms) with planetary origin were never determined in Kinoshita \& Souchay (1990), Souchay \& Kinoshita (1997), or REN2000.

We consider it proper to recall briefly the terminology and concepts relative to the Poisson and Oppolzer terms, as they are not easily visible when a solution for a non-rigid-Earth model is computed through a transfer function method. It is well known that nutations affect different axes, namely the angular momentum, figure, and rotation axes. The nutations of each pair of those axes are related to each other by adding certain terms known generically as Oppolzer terms (see, e.g., Dehant \& Capitaine 1996, for more details). Even though special attention was paid to the nutations of the axis of rotation in the earlier theories, before adopting IAU1980, now the interest is focused on the nutations of the angular momentum and the figure axes, understood as the orientation of those axes with respect to a celestial frame, assumed to be almost inertial or fixed in the space in the theoretical framework. The nutation of the figure axis is the one actually observed by the Very Long Baseline Interferometry (VLBI) technique (Gross 1992), while the nutations of the angular momentum vector, usually named Poisson terms by Kinoshita, are not observable. These last nutations do not depend on the rheological Earth model at the first order and are thus identical for rigid and non-rigid-Earth models. Conversely, the nutations of the figure axis depend on the considered Earth model, and they are obtained by adding to the Poisson terms the so-called Oppolzer terms for the figure axis. Further details for the rigid case, approached through the Hamiltonian formalism, can be found in Kinoshita (1977).

In the Hamiltonian formulation of the non-rigid-Earth theory by Getino \& Ferrándiz, the nutations of the figure axis are also given by the sum of the Poisson and Oppolzer terms; the latter depend on the geophysical model and their determination is rather more complicated in the non-rigid than in the rigid case. However, the application of a transfer function to a rigid Earth solution does not require the explicit computation of the Oppolzer terms for the non-rigid Earth to substitute the Oppolzer terms of the rigid model in the summation with the Poisson terms. The transfer function acts on the amplitudes of the rigid nutations expressed in their prograde and retrograde components, but the mechanism of amplification of the

\footnotetext{
2 ftp://maia.usno.navy.mil/conventions/archive/2003/ chapter5/tab5.3b.txt
}

amplitudes of solely their Oppolzer constituents (Poisson constituents are invariable) may be unclear although known from theory. For instance, in spite of its complexity and insight into the geophysical details, the MHB2000 theory did not provide any specific information on the magnitude of the non-rigid Oppolzer terms, which remained implicit after the application of the transfer function.

On the one hand, the specific reasons that led to the nonapplication of the transfer function to the whole REN2000 theory, but only to the direct lunisolar part of the solution, have never been published as far as we know, but we can infer reasonably that the main reason was that those terms were believed to be smaller than $5 \mu$ as according to the comments reproduced before. On the other hand, the truncation level of $5 \mu$ as was consistent with the results of the VLBI data analysis associated to MHB2000, as it corresponds to the formal error of most of the short-period terms from a total of the 21 frequencies considered in the amplitude estimations (Herring et al. 2002). Let us notice that the missing convolution could have been done without any problem, since for example, another contemporary solution, SF2000 (Shirai \& Fukushima 2001), applied a simpler transfer function based on Sasao et al. (1980) to the rigid-Earth solution RDAN97 (Roosbeek \& Dehant 1998a), and provided 32 planetary terms for a (non-rigid) two-layer Earth model.

It may not be out of place to recall that the rigid-Earth RDAN97 theory does not give separately the (direct) lunisolar and the indirect and direct planetary nutations, nor does it indicate the body that originates each term. However, the full set of planetary terms can be easily identified since they contain among their arguments the longitude of some planets different from the Earth. This allows us to identify as planetary 361 out of 959 terms of the main solutions to the obliquity nutations, gathered in the file DEPS98-main.txt (Roosbeek \& Dehant 1998b). For the longitude, 634 out of 1489 main terms are identified as planetary in the file DPSIS98-main.txt. That solution also gives explicit values for the Oppolzer terms for the figure and rotation axes corresponding to each period. It can be checked that all the Oppolzer terms corresponding to the planetary ones have zero amplitude. That fact is in accordance with our own results for the rigid Earth commented on in the next section. Finally let us recall that a partial computation of the Oppolzer terms associated with RDAN97 was published by Frede \& Dehant (1999), although the computation only included the main nine terms (none of planetary origin) and put the stress on the comparison of two methods, not in the obtaining of the full set of non-rigid nutations.

Conversely, planetary nutations for the figure axis of a nonrigid-Earth model (of three layers) were included in the GF2000 (Getino \& Ferrándiz 2000) solution derived by the very different Hamiltonian approach. The full series GF2000 and accompanying routines were only distributed inside the IAU working group on non-rigid-Earth nutations (Dehant 2002) and only a previous version addressing the direct lunisolar effects for a two-layer Earth model was published (Getino \& Ferrándiz 2001).

Nevertheless, although the planetary Oppolzer terms are indeed smaller than $5 \mu$ as in the rigid case, we show in this paper that the same does not happen in the non-rigid case: some of the terms have amplitudes well above the assumed accuracy threshold, which can even exceed $10 \mu$ as. Therefore, the lack of those terms is not just an issue of consistency, but is an avoidable uncertainty source that can no longer be ignored considering the current accuracy demands. The amplification of these terms is mainly explained by the resonance due to the free core nutation (FCN) as occurs in the main lunisolar part of the model, that is, 
excluding the planetary perturbations of the Moon and Sun orbits. The careful estimation of the associated deficiencies, which are the origin of deviations or systematic errors in the model, is presented in the next section.

\section{Computation of Oppolzer terms in the planetary nutations}

In order to study the rotation of the non-rigid Earth, we apply the Hamiltonian approach introduced by Getino \& Ferrándiz (1995). In this theory, both direct and indirect planetary effects are included, the former corresponding to the direct gravitational attraction of planets acting on the Earth's bulge and the latter resulting from the planetary perturbations of the orbits of the Moon and Sun. The derivation of the Oppolzer terms associated to those planetary perturbations is similar to that of the analogous terms due to the main lunisolar perturbations. The analytical expressions are actually formally identical, with slight changes. For instance, the Kinoshita-like constants $k_{\mathrm{M}}$ and $k_{\odot}$ are replaced by similar constants corresponding to each planet (for the direct effects) and the Kinoshita's coefficients $A^{(0)}, A^{(1)}$, and $A^{(2)}$, which depend on the orbits of the Moon and Sun, are substituted by the similar expansions in spherical harmonics of the relevant disturbing body. Of course, the strict association between in-phase and out-of-phase terms disappears from the different developments, but this fact does not imply any intrinsic difficulty, and the form of the expressions can be preserved by a simple introduction of an additional phase in the angular arguments of the expansions.

Let us start by recalling the formulae for the Poisson and Oppolzer terms due to the zonal component $(2,0)$ of the direct lunisolar potential in the case of a rigid Earth. We employ the same notation used in Getino \& Ferrándiz (1995) and Ferrándiz et al. (2016), similar to the original notation used by Kinoshita (1977). For the sake of conciseness, our notation is not explained here in detail, since the main difference with respect to Kinoshita's one lies in the notation of the Andoyer variables as $(\lambda, \mu, v, \Lambda, M, N)$ instead of $(h, g, l, H, G, L)$, and the use of $\sigma$ instead of $J$ for the colatitude of the angular momentum axis.

In this context, the Poisson terms raised by a perturbing body $B_{\mathrm{p}}$ of Kinoshita's constant $K_{\mathrm{p}}$ can be written (Getino \& Ferrándiz 1995; Ferrándiz et al. 2016) as

$\Delta \lambda=-K_{\mathrm{p}} \frac{1}{\sin I} \frac{\partial W_{\mathrm{a}}}{\partial I}=-\frac{K_{0}}{\sin I} \sum_{i} \frac{\partial}{\partial I}\left(\frac{B_{i}}{n_{i}}\right) \sin \Theta_{i}$,
$\Delta I=K_{\mathrm{p}} \frac{1}{\sin I} \frac{\partial W_{\mathrm{a}}}{\partial \lambda}=\frac{K_{0}}{\sin I} \sum_{i}\left(-m_{5}\right) \frac{B_{i}}{n_{i}} \cos \Theta_{i}$,

and the Oppolzer terms are given by

$$
\begin{aligned}
& \Delta\left(\lambda_{\mathrm{f}}-\lambda\right)=K_{\mathrm{p}} \frac{1}{\sin I} \sum_{\tau= \pm 1} \sum_{i} \frac{\tau C_{i}(\tau)}{n_{\mu}-\tau n_{i}} \sin \Theta_{i}, \\
& \Delta\left(I_{\mathrm{f}}-I\right)=K_{\mathrm{p}} \sum_{\tau= \pm 1} \sum_{i} \frac{C_{i}(\tau)}{n_{\mu}-\tau n_{i}} \cos \Theta_{i} .
\end{aligned}
$$

As we recalled above, the Oppolzer terms provide the nutations of the figure axis, $\lambda_{\mathrm{f}}$ and $I_{\mathrm{f}}$, with respect to that of the angular momentum axis, $\lambda$ and $I$ (both angles reckoned with opposite sign with respect to the longitude $\psi$ and obliquity $\epsilon$ used in the equinox based paradigm).

If we consider an inelastic two-layer Earth model with dissipation at the core-mantle boundary, the Oppolzer terms in longitude and obliquity can be calculated as a sum of two components given by the following equations that extend the former (Getino \& Ferrándiz 2001),

$$
\begin{aligned}
\Delta_{0}\left(\psi_{\mathrm{f}}-\psi\right) & =-K_{\mathrm{p}} \frac{1}{\sin I} \sum_{i, \tau= \pm 1} C_{i}(\tau)\left[F_{1}^{a} \tau \sin \Theta_{i}-F_{1}^{b} \cos \Theta_{i}\right] \\
\Delta_{0}\left(\epsilon_{\mathrm{f}}-\epsilon\right) & =-K_{\mathrm{p}} \sum_{i, \tau= \pm 1} C_{i}(\tau)\left[F_{1}^{a} \cos \Theta_{i}+F_{1}^{b} \tau \sin \Theta_{i}\right]
\end{aligned}
$$

and

$$
\begin{aligned}
\Delta_{t}\left(\psi_{\mathrm{f}}-\psi\right) & =-\frac{1}{\sin I} \sum_{i, \tau= \pm 1} C_{i}(\tau)\left[E_{1} \tau \sin \Theta_{i}-E_{2} \cos \Theta_{i}\right] \\
\Delta_{t}\left(\epsilon_{\mathrm{f}}-\epsilon\right) & =-\sum_{i, \tau= \pm 1} C_{i}(\tau)\left[E_{1} \cos \Theta_{i}+E_{2} \tau \sin \Theta_{i}\right]
\end{aligned}
$$

in terms of functions $F_{1}^{\mathrm{a}}, F_{1}^{\mathrm{b}}, E_{1}$, and $E_{2}$ described in Getino \& Ferrándiz (2001). The first pair extends the rigid solution to Poincaré's model - with mantle and liquid core - whilst Eq. (4) gather the effects of deformations.

For the numerical evaluation of the previous expressions, unlike in the MHB2000 theory, we do not adopt the coefficients obtained by Kinoshita \& Souchay (1990), where the planetary expansions came from ELP2000 (Chapront-Touzé \& Chapront 1983b) and the old theory VSOP82 (Bretagnon 1982). Instead, the expansions in spherical harmonics have been computed via a special purpose-built algebraic processor that handles the so-called Kinoshita series (Navarro \& Ferrándiz 2002; Navarro 2002), using as inputs the semi-analytical orbital theories VSOP87 (Bretagnon 1988) and ELP2000-82B (Chapront-Touzé \& Chapront 1988). The values of the remaining parameters are those in Table 1 of Getino \& Ferrándiz (2001) in which the same orbital solutions were used.

The planetary Oppolzer terms for a rigid Earth model (though not computed in REN2000) can be easily obtained by evaluating Eq. (2) with certain parameters set to zero. It happens that the maximum amplitudes of individual contributions to longitude and obliquity only reach 1.1 and $0.4 \mu$ as, respectively, so that the amplitudes of each term are really smaller than $5 \mu$ as in the rigid case according to our calculations.

Non-rigid planetary Oppolzer terms given by Eqs. (3) and (4) were evaluated separately for the direct effects of Venus, Jupiter, and Saturn, and the indirect planetary effect on the orbits of the Moon and Sun. The terms with total amplitude larger than $10 \mu$ as are displayed in Table 1, showing their origin (together with the terms of less amplitude but identical argument). The nutation arguments are denoted in the standard form, as in the IERS Conventions (2010). We also provide them in the CIObased paradigm by giving the $\mathrm{d} X$ and $\mathrm{d} Y$ offsets of the Celestial Intermediate Pole (CIO) or CPO.

\section{Discussion and conclusions}

As shown in the previous section, the rigorous computation of the Oppolzer terms for the non-rigid-Earth model reveals that their amplitudes are not negligible at all. A few of them exceed the threshold of $5 \mu$ as indicated in the computation programs of IAU2000A to justify the omission of the Oppolzer terms; moreover, the term with a period of 398.884 mean solar days is far above that boundary, since it reaches almost $50 \mu$ as in longitude and $20 \mu$ as in obliquity.

These quantities may seem small but they are not when compared to the whole set of planetary terms of IAU2000A. On the 
Table 1. Largest Oppolzer terms of planetary origin for the Earth's figure axis.

\begin{tabular}{rrrrrrrrrrrrrrr}
\hline \hline$L_{\mathrm{Ve}}$ & $L_{\mathrm{E}}$ & $L_{\mathrm{Ma}}$ & $L_{\mathrm{J}}$ & $p_{\mathrm{A}}$ & $\begin{array}{r}\text { Period } \\
(\text { days })\end{array}$ & $\begin{array}{r}\Delta \psi_{\text {in }} \\
(\sin )\end{array}$ & $\begin{array}{r}\Delta \psi_{\text {out }} \\
(\cos )\end{array}$ & $\begin{array}{r}\Delta \epsilon_{\text {in }} \\
(\cos )\end{array}$ & $\begin{array}{r}\Delta \epsilon_{\text {out }} \\
(\sin )\end{array}$ & $\begin{array}{r}\mathrm{d} X_{\mathrm{in}} \\
(\sin )\end{array}$ & $\begin{array}{r}\mathrm{d} X_{\text {out }} \\
(\cos )\end{array}$ & $\begin{array}{r}\mathrm{d} Y_{\text {in }} \\
(\cos )\end{array}$ & $\begin{array}{r}\mathrm{d} Y_{\text {out }} \\
(\sin )\end{array}$ & $\begin{array}{r}\text { Origin } \\
\text { code }\end{array}$ \\
\hline 0 & 1 & 0 & -1 & 0 & 398.884 & 1 & 0 & 0 & 0 & 0.2 & 0 & 0.2 & 0 & 0 \\
2 & -4 & 0 & 0 & 2 & -487.638 & -4 & 0 & 2 & 0 & -1.8 & -0.1 & 1.7 & -0.1 & 1 \\
0 & 1 & 0 & -2 & 0 & 439.332 & 3 & -35 & 1 & 14 & 1.1 & -14 & 1.3 & 14.3 & 1 \\
0 & 3 & -4 & 0 & 0 & 418.266 & -8 & 1 & -3 & 0 & -3.2 & 0.5 & -3.1 & -0.4 & 1 \\
3 & -4 & 0 & 0 & 0 & 416.688 & -7 & 21 & -3 & -8 & -2.9 & 8.2 & -2.9 & -7.9 & 1 \\
0 & 1 & 0 & -1 & 0 & 398.884 & -47 & -8 & -17 & 3 & -18.8 & -3 & -17.2 & 3 & 1 \\
0 & 2 & -2 & 0 & 0 & 389.968 & -11 & -1 & -4 & 0 & -4.3 & -0.4 & -3.8 & 0.4 & 1 \\
2 & -4 & 0 & 0 & 2 & -487.638 & -14 & -1 & 6 & -1 & -5.6 & -0.5 & 5.6 & -0.5 & 2 \\
0 & 1 & 0 & -1 & 0 & 398.884 & 4 & 1 & 1 & 0 & 1.6 & 0.2 & 1.5 & -0.2 & 5 \\
\hline
\end{tabular}

Notes. Units: amplitudes in $\mu$ as, periods in mean solar days. The IERS fundamental nutation arguments $l, l^{\prime}, F, D, \Omega, L_{\mathrm{Me}}, L_{\mathrm{Sa}}, L_{\mathrm{U}}$, and $L_{\mathrm{Ne}}$ have 0 coefficients and are not shown. Effect origin code: 0 indirect Moon; 1 indirect Sun; 2 direct Venus; 5 direct Jupiter. $\Delta \psi \& \Delta \epsilon$ are displayed as integers as in the original MHB2000 routine.

one hand, only 15 out of the 687 terms have amplitudes larger than $50 \mu$ as in the longitude series, and only 11 terms have amplitudes larger than $20 \mu$ as in obliquity; besides, the mean is $3.8 \mu$ as, the median only $0.5 \mu$ as, and 598 terms have amplitudes lower than precisely $5 \mu$ as. Nowadays, a much larger set of amplitudes can be determined from VLBI observations (Belda et al. 2017) and their formal errors accumulate nearly $2 \mu$ as (Gattano et al. 2017; Malkin 2014).

The amplification of the Oppolzer terms with respect to the corresponding terms in the rigid Earth model is due to the effect of the resonance associated to the Earth's retrograde nearly diurnal free wobble (NDFW) - responsible for the free core nutation $(\mathrm{FCN})$ - on the forced nutations with nearby periods, as happens with the first order lunisolar nutations. A recent determination from VLBI and solid tides data by Krasna et al. (2013) found 430.00 mean solar days for the FCN period, close to the Lambert's value (Lambert 2007) recommended in the IERS Conventions (2010). As shown in Table 1, the largest effects occur at the periods $398.884,416.688,439.332$, and -487.638 days $^{3}$.

Since this is the first computation of the Oppolzer terms of planetary origin, there is no previous solution to check our results directly and the validation has to be based on indirect evidence. The periods also appear in the REN2000 series ${ }^{4}$. More relevant is that differences of similar magnitude in the amplitudes of those terms appear between SF2000 and RDAN97 solutions, which we can attribute mainly to the amplification of the Oppolzer terms, hidden because of the use of a transfer function method.

A definitive validation of the correction of our results should be based on tests with observational data. The lack of nonnegligible terms should give rise to deviations and systematic errors in the nutation model, which should be apparent in the residuals. Therefore, we have first checked the impact on the variance of the offsets $\mathrm{d} X$ and $\mathrm{d} Y$ time series given in the last conventional IERS solution EOP 14 C04 (Bizouard et al. 2017, 2018). We have disregarded the first years of VLBI data due to their poorer quality and used a time span of 28 years, 1990-2017. The CPO values have been weighted in the customary way, with the inverse square formal errors given in the series. We have compared the weighted root mean square (WRMS) of the $\mathrm{d} X$

\footnotetext{
The sign is irrelevant since no decomposition is made in pro- and retrograde motions.

4 Available at ftp://syrte9. obspm. fr/REN2000/ and can be identified in the much longer RATGP95 expansion (Roosbeek 1996).
}

Table 2. Variation of WRMS in period 1990-2017 depending on CPO correction for missing planetary Oppolzer terms.

\begin{tabular}{lrr}
\hline \hline \multirow{2}{*}{ Data source } & \multicolumn{2}{c}{ WRMS } \\
\cline { 2 - 3 } EOP 14 C04 & \multicolumn{1}{c}{$\mathrm{d} X$} & \multicolumn{1}{c}{$\mathrm{d} Y$} \\
\hline Without correction & 165.5 & 171.9 \\
With correction & 159.1 & 167.1 \\
WRMS decrease & 6.4 & 4.8 \\
\hline
\end{tabular}

Notes. Units are in $\mu$ as.

and $\mathrm{d} Y$ series with the WRMS of them after applying the correction corresponding to the missing planetary Oppolzer terms. The results are shown in Table 2. Roughly speaking, the WRMS decreases nearly $5 \mu$ as when the neglected planetary Oppolzer terms are taking into account to explain the CPO variability.

However, the refinement of the calculated amplitudes of the investigated terms by VLBI data analysis is not simple, due to the concurrence of the FCN and the well-known difficulty in separating the true FCN signal from the missing terms.

For instance, the largest systematic error $(40 \mu$ as in $\Delta \psi)$ associated to the omission of the new Oppolzer term of a period of 398.884 days recalls the unusually large value ( $23 \mu \mathrm{as})$ of the formal error in the term of the nearby period ( -386.00 days) given in Table 1 of Herring et al. (2002). Besides, the new terms with periods between 400 and 500 days will affect the analysis performed in frequency bands near to the FCN, as shown in Figs. 3 and 4 in Malkin \& Miller (2007).

The empirical estimation of the amplitudes of the missing Oppolzer terms is not fully independent of any empirical FCN model obtained by fitting its time varying amplitude and phase to a time series of observed nutations, usually by means of a sliding window approach (Malkin 2014; Belda et al. 2016). Therefore, neither the relationship between the addition of the corrections to planetary nutations nor the diminution of the root mean square of residuals can be a simple issue, and interactions with the accompanying FCN model are expected.

In an attempt at circumventing the difficulties arising from the FCN dependence, we have considered the possibility of performing additional tests by taking advantage of an existing alternative approach, in which the parameters of a VLBI global solution are expressed in the polar motion form and the three Earth orientation parameters (EOP) (excluding CPO) are subject to a Fourier analysis (Petrov 2007). Empirical amplitudes are determined for a wide set of frequencies (in the terrestrial frame) 


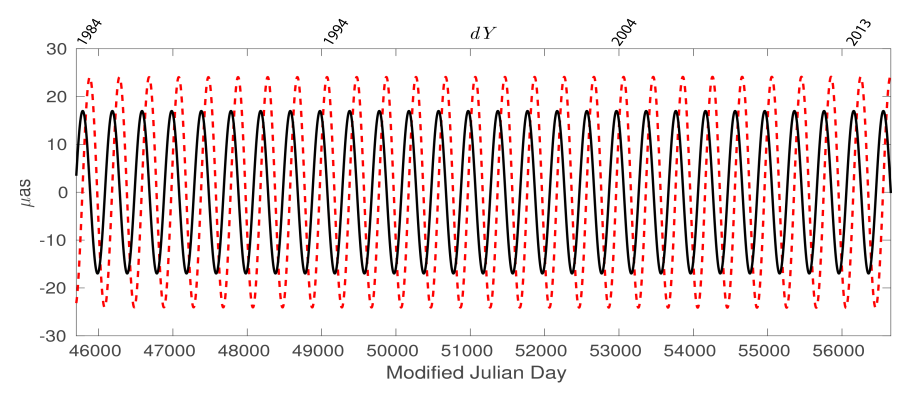

Fig. 1. Comparison of largest harmonic component $\mathrm{d} Y$ predicted with our model, with period $\pm 398.884 \mathrm{~d}$ (in black), and two constituents of Petrov's VLBI solution with closest periods specified in the text (in red). The time span is 1984-2013.

chosen to get a maximum separation of components. Frequencies are not necessarily linked to a physical excitation, but the procedure allows us to provide a good approximation of nutations. The spectrum in Petrov's VLBI solution heo_22c for the period $1984-2013^{5}$ includes a couple of nearly diurnal frequencies $\left(-73.10347232\right.$ and $\left.-72.73884479 \mu \mathrm{rad} \mathrm{s}^{-1}\right)$ whose periods in the celestial frame differ from \pm 398.884 days in less than \pm 0.05 days. The harmonic content of this pair is slightly larger compared to that of our corresponding Oppolzer term; a comparison of the $\mathrm{d} Y$ component of the CPO computed from each model in the time domain is displayed in Fig. 1; the result for $\mathrm{d} X$ is similar. We consider that all the former evidence gives good support to our analytical solution.

The theoretically inherent inconsistency of the geophysical models used in the lunisolar (three-layers, oceans, anelasticity) and planetary (rigid) portions of the IAU2000 model does affect its accuracy as shown and thus it is not irrelevant; accordingly, the planetary nutation theory must be updated to account for the Earth's non-rigidity.

The full revision of the theory of planetary nutations is not a straightforward task for several reasons, including the improvements of the solutions to the orbital motion of Moon and planets and the updates of the astronomical standards in the last decades. In the mean time, accuracy and consistency may be improved by using corrections to account for the missing terms, as done before with the corrections for consistency between IAU2000 and IAU2006 precession theory (Capitaine et al. 2003, 2005), which are recommended in the IERS Conventions (2010). More recent corrections for improving the dynamical consistency between the former two IAU models have been proposed (Escapa et al. 2017) and the possibilities of their application was a matter under consideration (Escapa \& Capitaine 2018).

There exists some agreement towards the need for adopting corrections to improve the accuracy and consistency of the EOP models. The proposal of both new EOP models and corrections to existing models is among the expected outputs of the IAU/International Association of Geodesy Joint Working Group on Theory of Earth rotation and validation (IAU/IAG JWG TERV) currently in operation (Ferrándiz \& Gross 2015; Huang et al. 2015).

The Oppolzer terms presented here may be thought to be not fully consistent with the IAU2000 due to the use of some different basic Earth parameters in MHB2000 and this approach. However, the variation of the final figures is not expected to reach $4 \%$ even in a pessimistic estimation. Therefore, the incorporation of corrections to the IAU2000 model corresponding to the missing planetary Oppolzer terms is a feasible option, in our opinion, which can be implemented in the short- to midterm if so agreed.

Acknowledgements. The authors thank the anonymous referee for his/her valuable comments and suggestions that helped to improve the manuscript. This work was partly supported by grant AYA2016-79775-P (AEI/FEDER, UE).

\section{References}

Belda, S., Ferrándiz, J. M., Heinkelmann, R., Nilsson, T., \& Schuh, H. 2016 , J. Geodyn., 94, 59

Belda, S., Heinkelmann, R., Ferrándiz, J. M., et al. 2017, AJ, 154, 166

Bizouard, C., Lambert, S., Becker, O., \& Richard, J. Y. 2017, IAG Symp., 134, 265

Bizouard, C., Lambert, S., Becker, O., \& Richard, J. Y. 2018, in The IERS EOP C04 Solution Consistent with ITRF2014, Proc. Journées 2017, des Systèmes de Référence et de la Rotation Terrestre: Furthering our Knowledge of Earth Rotation, in press

Bretagnon, P. 1982, A\&A, 114, 278

Bretagnon, P. 1988, A\&A, 202, 304

Capitaine, N., Wallace, P. T., \& Chapront, J. 2003, A\&A, 412, 567

Capitaine, N., Wallace, P. T., \& Chapront, J. 2005, A\&A, 432, 355

Chapront-Touzé, M., \& Chapront, J. 1983a, A\&A, 91, 233

Chapront-Touzé, M., \& Chapront, J. 1983b, A\&A, 124, 50

Chapront-Touzé, M., \& Chapront, J. 1988, A\&A, 190, 342

Dehant, V. 2002, Highlights of Astronomy, ed. H. Rickman, 12, 117

Dehant, V., \& Capitaine, N. 1996, Celest. Mech. Dyn. Astron., 65, 239

Escapa, A., \& Capitaine, C. 2018, in Proc. Journées 2017, des Systèmes de Référence et de la Rotation Terrestre: Furthering our Knowledge of Earth Rotation, in press

Escapa, A., Getino, J., Ferrándiz, J. M., \& Baenas, T. 2017, A\&A, 604, A92

Ferrándiz, J. M., \& Gross, R. S. 2015, Int. Assoc. Geodesy Symp., 143, 533

Ferrándiz, J. M., Navarro, J. F., Escapa, A., \& Getino, J. 2016, Pure Appl. Geophys., 172, 57

Frede, V., \& Dehant, V. 1999, J. Geod., 73, 94

Gattano, C., Lambert, S., \& Bizouard, C. 2017, J. Geod., 91, 849

Getino, J., \& Ferrándiz, J. M. 1995, Celes. Mech., 61, 117

Getino, J., \& Ferrándiz, J. M. 2000, IAU Colloq., 180, 236

Getino, J., \& Ferrándiz, J. M. 2001, MNRAS, 322, 785

Gross, R. S. 1992, Geophys. J. Int., 109, 162

Herring, T. A., Mathews, P. M., \& Buffet, B. A. 2002, J. Geophys. Res., 107, 2069

Hohenkerk, C., \& The IAU SOFA Board 2010, SOFA Tools for Earth Attitude, IAU (Available at http: //www. iausofa.org)

Huang, C., Gross, R., Seitz, F., et al. 2015, IAU Proc., 11, 60

IERS Conventions 2010, IERS Technical Note, eds. G. Petit, \& B. Luzum, 36

Kaplan, G. 2005, US Naval Observatory Circulars, 179

Kinoshita, H. 1977, Celest. Mech. Dyn. Astron., 15, 277

Kinoshita, H., \& Souchay, J. 1990, Celest. Mech. Dyn. Astron., 48, 187

Krásná, H., Böhm, J., \& Schuh, H. 2013, A\&A, 555, A29

Lambert, S. 2007, Technical Note (Available at http://syrte.obspm.fr)

Malkin, Z. M. 2014, Astron. Rep., 58, 415

Malkin, Z. M., \& Miller. 2007, Proc. 18th European VLBI for Geodesy and Astrometry Work Meeting (Technische Universität Wien), 93

Mathews, P. M., Herring, T. A., \& Buffet, B. A. 2002, J. Geophys. Res., 107, 2068

Navarro, J. F. 2002, Doctoral Dissertation (in Spanish), Universidad de Alicante, Spain

Navarro, J. F., \& Ferrándiz, J. M. 2002, Celest. Mech. Dyn. Astron., 82, 243

Petrov, L. 2007, A\&A, 467, 359

Roosbeek, F. 1996, Geophys. J. Int., 126, 197

Roosbeek, F., \& Dehant, V. 1998a, Celest. Mech. Dyn. Astron., 70, 215

Roosbeek, F., \& Dehant, V. 1998b, RDAN98: Technical Note (available from the authors)

Sasao, T., Okubo, S., \& Saito, M. 1980, Proc. IAU Symp. 78, 165

Shirai, T., \& Fukushima, T. 2001, AJ, 121, 3270

Souchay, J., \& Kinoshita, H. 1997, A\&A, 318, 639

Souchay, J., Losley, B., Kinoshita, H., \& Folgueira, M. 1999, A\&AS, 135, 111

5 http://astrogeo.org/petrov/discussion/heo_22c/ 